\title{
A Method of Antarctic Temperature Forecasting Based on Time Series Model
}

\author{
Liwen Liang \\ North China Electric Power University, Baoding, Hebei 071000, China \\ 1325578456@qq.com
}

Key words: Antarctic Temperature Forecasting, Correlation Analysis, Linear regression analysis, Time series analysis

\begin{abstract}
Based on the analysis of the Antarctic temperature change trend, this paper analyze the influence factors of the surface temperature in Antarctica, and established the corresponding model. Then, it extracts a part of the time series of dynamic data part provided by the site, and drew a timing diagram of sample data and some images about autocorrelation and partial correlation, correlation analysis.
\end{abstract}

\section{Introduction}

Environmental problem has been an important issue in the process of the development of human society. In recent years, the emissions of greenhouse gases have caused a series of environmental problems, including global warming. The study found that the middle and high latitude climate change is more obvious than lower latitudes. At the same time, human beings have been concerned about the influence of the change of temperature in Antarctic, ice conditions and atmospheric circulation.

Antarctica is one of the world's largest temperature gradient, and is also a sensitive point in the global climate change. The change of temperature in Antarctica has a significant impact on the climate and atmospheric circulation of the southern hemisphere, and also affects the climate change in the northern hemisphere. Therefore, the research on the temperature change of the Antarctic surface is of great significance.

\section{Basic Assumption}

-Do not consider the impact of extreme weather on the average surface temperature, less precipitation, snow may make the temperature of Antarctica lower.

- Assume that the same latitude regions of Antarctica would be able to receive the same solar heat.

- Ignore the difference between the average surface temperature of the warm and cold seasons in Antarctica during the two seasons.

-Ignore the difference of the ocean on the average surface temperature in the presence of Antarctica.

-Ignore the impact of a small amount of vegetation on the temperature in Antarctica.

\section{The model}

In view of the above problem, we select the surface temperature data in a certain period of time, to establish the following function

$$
\mathrm{T}=\mathrm{f}(\mathrm{La}, \mathrm{Lo}, \mathrm{He}, \mathrm{PS}, \mathrm{Pa})
$$

\subsection{Latitude}

Due to the higher latitude, the smaller the altitude of the sun, the path of the solar radiation through the atmosphere is longer, and the solar radiation weakened by the atmosphere is stronger, so as to reach the ground less solar radiation, so the temperature is low. Therefore, we will transform the analysis of latitude to the analysis of solar radiation. 
Surface of Antarctica receiving the external environment mainly comes from the sun's radiation, but the sun's radiation, mainly in the daytime, the amount of radiation varies with the seasons, times, weather, and geographical conditions.

\subsection{Height}

Because the troposphere (located in the lowest layer of the atmosphere, focused about $75 \%$ of the atmosphere and the quality of more than $90 \%$ of the water vapor quality. The lower into the ground, the upper bound height change with latitude and season, the polar region average $8 \sim 9 \mathrm{~km}$.)

Atmosphere is the main direct heat source of the ground, the farther from the ground, the less from the radiation surface, the temperature will be low. And every $100 \mathrm{~m}$, the temperature drops of 0.6 degrees Celsius (vertical lapse rate). So the higher the altitude, the lower the temperature.

\subsection{Atmospheric circulation}

Atmospheric circulation refers to the movement of atmospheric circulation in a global scale around the earth, with a certain time scale and spatial scales. Its horizontal scale is above more than thousands of kilometers, vertical scale more than $10 \mathrm{~km}$ and the time scale over several days. Globally, atmospheric circulation realizes the water heat exchange between high and low latitude, and sea and land, which is an important factor in forming the weather and climate around the world.

Movement of atmospheric circulation, carrying the moisture and heat over the ocean, make the water circulate in the ocean and between the land and sea. Antarctica is located in the middle and high latitude, hence the polar circulation is the main reason of its weather. The scope of activities of polar circulation is within the troposphere limitedly, the highest just up to the roof equivalent to 8000 meters. When the air reaches the Arctic range, temperature sharply lows, and it is cold and dry, by the Coriolis force deflection influence westward, forming polar easterlies, therefore the surface temperatures of the Antarctic region is low, year-round cold.

\subsection{Land sea difference}

The ability of oceans to absorb and store energy is different from continents. Antarctic land area is big, the heat storage ability is weak, warm heat radiation out soon. Although the water heat capacity is big, can absorb more heat, and heat is slower. But Antarctica land area is far less than sea area, thus caused the Antarctic cold all the year round, the annual average temperature is - 56 degrees Celsius.

\section{Based on time series prediction: ARIMA model}

ARMA prediction method is composed of free regression model (AR) and moving average model (MA), and ARMA system is regarded as a random sequence of data sequence which is formed by the object over time. Although the single sequence of the sequence is uncertain, the whole sequence is changed regularly. If the time series is regarded as a non - stationary sequence, the difference can be achieved. According to the characteristic of system by several methods of cross use should be in the practical application, and then select the appropriate order $(\mathrm{P}, \mathrm{q})$ as a model to be built.

We can determine a certain range of the optimal model order, namely $p=2$, $q=1$ by self-correlation and partial correlation graph, so we choose the model ARIMA $(2,1)$ to deal with the image of the site Rethera data fitting as follows: 


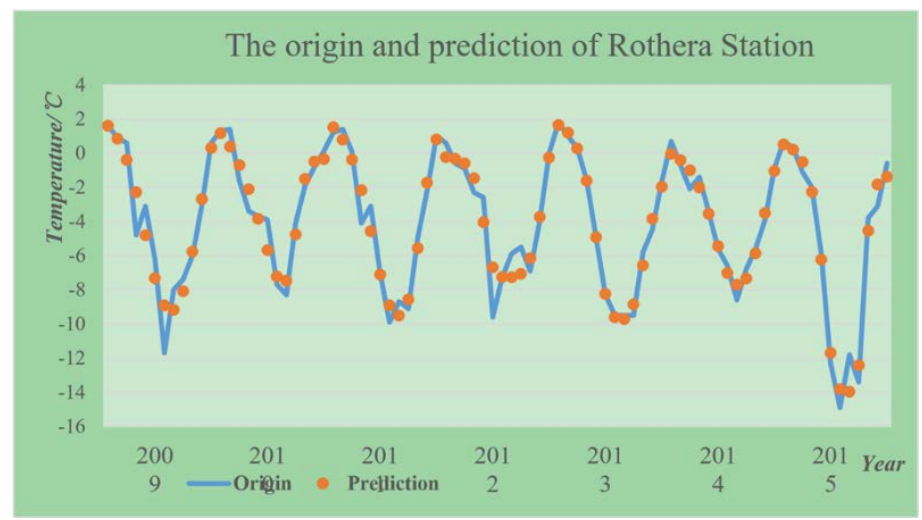

Figure 1. The prediction of Rethera Station

We also use time series model and MATLAB to describe the other four sites: Station Grytviken, Station Davis, Station Sanmartin and Station Vostok original image and predictive image as shown.
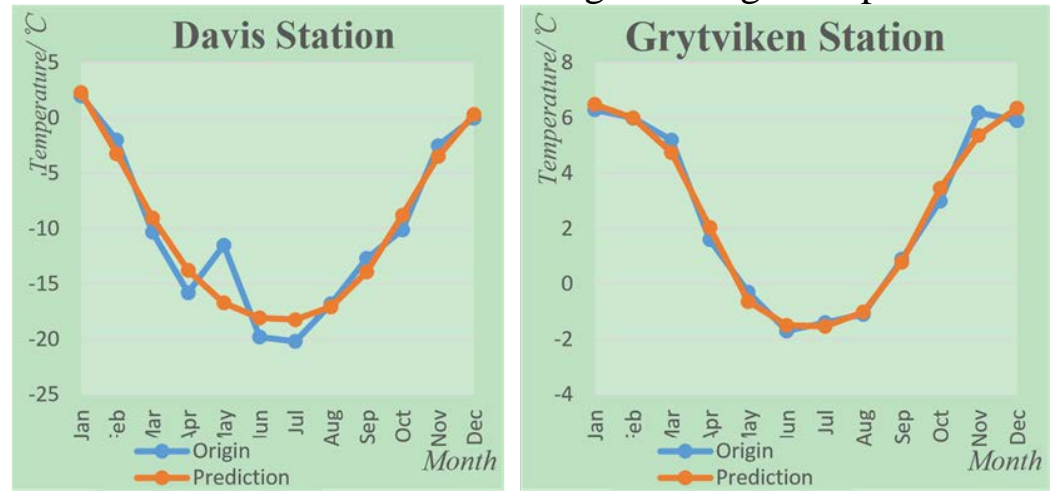

Figure 2. Davis Station and Grytviken Station
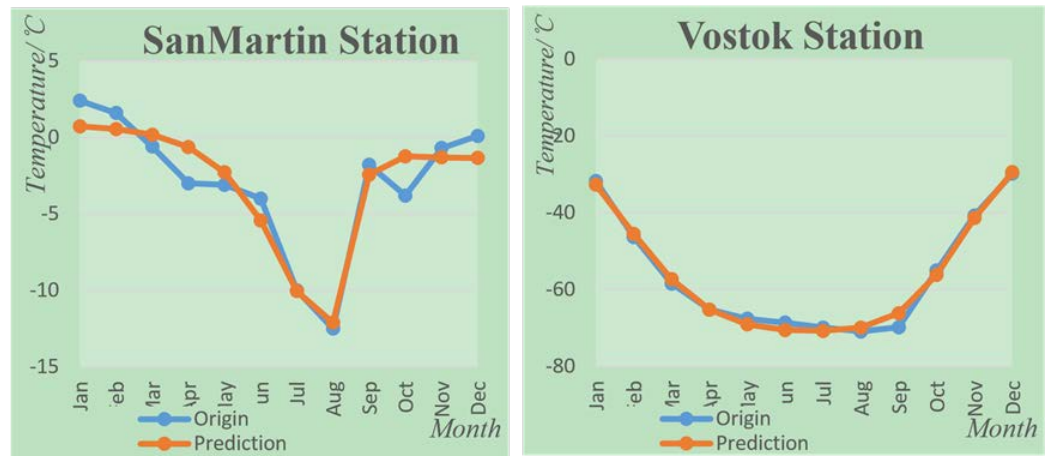

Figure 3. SanMartin Station and Vostok Station

\section{Sensitivity Analysis}

Through mathematical software, we achieved the sensitivity analysis of the three parameters of latitude, altitude, longitude. The model is very sensitive to latitude and altitude. It can reduce the influence of the external inevitable errors. The following are three images on the three parameters of sensitivity analysis: 


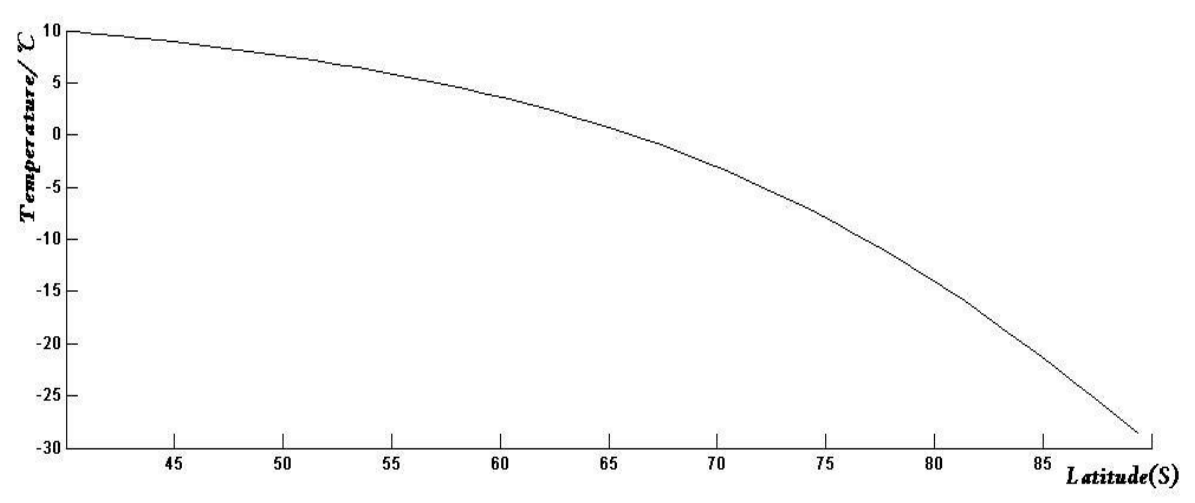

Figure 4. The influence of latitude on temperature

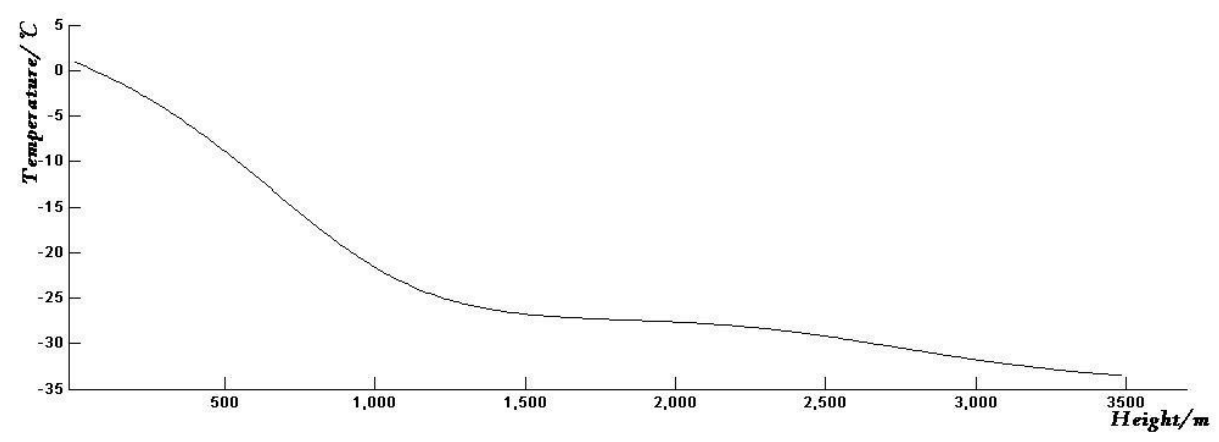

Figure 5. The influence of altitude of temperature

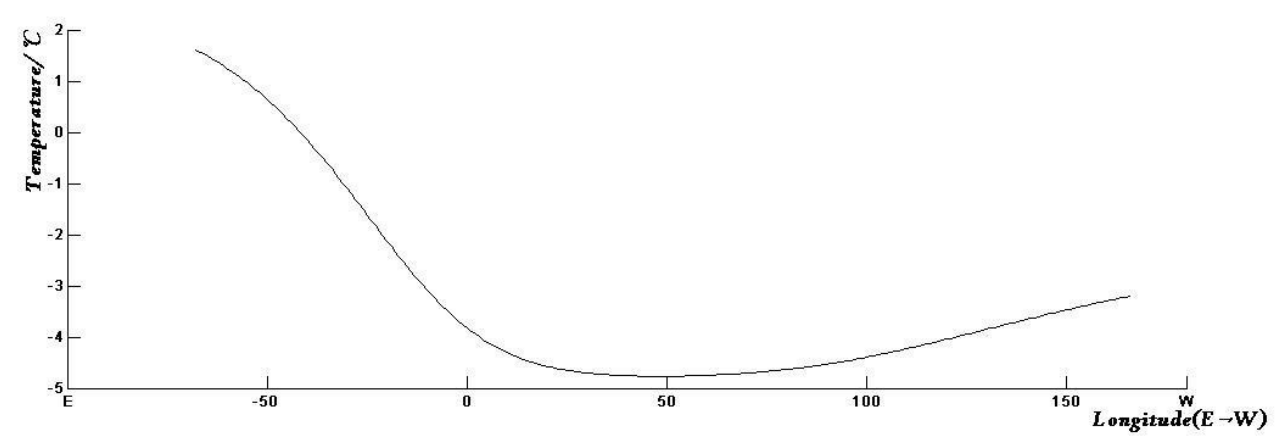

Figure 6. The influence of the longitude of temperature

This model is examined to be very robust with respect to parameter variation. Considering that the parameters of this model are results of last model, which are very stable, the optimal solution of this model will consequently be stable too.

\section{Summary}

We conducted sensitivity analyses on the latitude, longitudes and altitude. It can be seen that the main factors of temperature is latitude and altitude, nevertheless, there is nothing to with the longitude.

\section{References}

[1] Lv Xiangyin, Ling Yongshun, Huang Chaochao. Calculation of surface temperature and infrared radiation for ground target[A]. Anhui Province: Electronic Engineering Institute of PLA, 2005.

[1] Xiao Zixin, The research based on the time series analysis on the trend of the EUR against the RMB exchange rate fluctuations, Changsha: Central South University. 
[2] Jiang Qiyuan, The mathematical model (the Forth Edition ), Beijing: higher education press, 2011.

[3] Si Shoukui, Mathematical modeling algorithms and procedures(the Second Edition) (p474537), Beijing: National Defend Industry Press, 2011. 